\title{
PROBLEMS CONCERNING THE \\ EXTRAGALACTIC DISTANCE SCALE
}

\author{
G. O. ABELL \\ University of California, Los Angeles, Calif., U.S.A.
}

\begin{abstract}
The use of the brightest members of galaxy clusters as distance indicators is questioned because of a possible correlation between the absolute magnitude of the brightest galaxy and the richness of the cluster. Comparison of the clusters' luminosity functions is considered to be more appropriate. The difference between the two approaches is substantial when a comparison is made between the Coma and Virgo clusters. The value of the Hubble parameter may well be less than $50 \mathrm{~km} \mathrm{~s}^{-1} \mathrm{Mpc}^{-1}$.
\end{abstract}

\section{Introduction}

The most remote galaxies for which we can estimate distances from the observed properties of known kinds of objects in them - brightest stars, globular clusters, novae, Hil regions, or other resolved features - are those in the Virgo cluster. To determine the extragalactic distance scale, however - or, equivalently, the Hubble constant - we must measure redshifts of galaxies (or clusters of galaxies) of known distances that are great enough for us to have confidence that those redshifts are representative of the general redshift-distance relation.

Unfortunately, for at least two reasons we can not be sure that the radial velocities of the Virgo cluster galaxies do not contain significant components of redshift that are not due to the general expansion: (1) there is evidence (de Vaucouleurs, 1953, 1956, 1958; Carpenter, 1961; Abell, 1961) that the Virgo cluster may be part of a local supercluster of groups and clusters; if so, there is the possibility that gravitational interactions between the member clusters result in contributions to the observed radial velocities that at least partly mask the universal expansion redshifts we want to measure; (2) there is a difference of nearly $500 \mathrm{~km} \mathrm{~s}^{-1}$ between the means of the radial velocities of the spiral galaxies and of the elliptical and S0 galaxies in the cluster (Holmberg, 1962; de Vaucouleurs, 1961). Even if there are not accelerations between component members of a local supercluster, we must be concerned about either a complex structure of what appears to be 'the' Virgo cluster or anomalous velocities of its constituent parts. In any case, it is hardly the ideal object with which to calibrate the velocity-distance relation.

We must, therefore, consider redshifts of more remote clusters, such as A1656 in Coma Berenices or A2065 in Corona Borealis (the numbers are those in the Abell (1958) catalogue). The familiar indicators referred to above are unresolved in these clusters; hence we must estimate their distances by comparing their brighter galaxies to those comparatively nearby galaxies whose distances we can hope to determine by more direct means - in particular, galaxies in the Virgo cluster. 
Most of the best-studied remote clusters are those great aggregations whose member galaxies are nearly all of the E and S0 types (Abell, 1965). Because of the possibility that the spiral and elliptical galaxies in the Virgo cluster are not at the same distance, we should carry out the comparison, if possible, with the elliptical galaxies in the Virgo cluster, and not with the spirals.

Sandage (1968), recognizing this fact, estimates the distance to M87 - the secondbrightest $\mathrm{E}$ galaxy in the Virgo cluster (or first brightest according to the photometry of some investigators) - from Racine's (1968) photometry of 2000 globular clusters associated with that object. Sandage noted that if the brightest globular cluster in M87 ( $B=21.3$ according to Racine) is the same in intrinsic luminosity as the brightest in M31 $(B=15.01)$, the modulus of M87 is 31.1. The difficulty of photometry of the globular clusters in M87 is great, and the magnitudes obtained by Racine are, perhaps, not definitive. Moreover, as de Vaucouleurs (1970) has pointed out, there may well be a significant difference between the absolute magnitudes of the brightest globular clusters in M31 and M87, the latter of which has a greater number of clusters by an order of magnitude. We shall not, however, be concerned here with the actual modulus of the elliptical galaxies in the Virgo cluster, but shall, rather regard that quantity as still uncertain by at least $0.5 \mathrm{mag}$.

What we are concerned with here is the next step - namely, that of finding the relative distances of the Virgo cluster ellipticals and the more remote clusters whose redshifts (hopefully) are representative of the general expansion of the universe. As stated above, the determinations of these relative distances require comparisons of galaxies in the distant clusters to those in the Virgo cluster. Clearly such a comparison can yield correct relative distances only if it concerns intrinsically identical objects in the near and distant clusters. The point of this discussion is to consider how such intrinsically identical objects can be recognized.

\section{The Brightest Cluster Galaxy}

Sandage (1958) has shown that for the groups and clusters studied by him that contain conspicuous elliptical galaxies, a plot of the logarithms of the redshifts of those clusters against the magnitudes of their brightest elliptical galaxies (corrected for absorption and the $K$-effect) shows a dispersion of less than 0.3 mag., which leads him to adopt the apparent magnitude of the brightest elliptical galaxy in a cluster as a distance indicator. He calibrates the extragalactic distance scale, and thus obtains a value for the Hubble constant, with the absolute magnitude of the brightest galaxy in the Virgo cluster, which he presumes to be intrinsically similar to the mean of the brightest elliptical galaxies in other clusters (with an uncertainty, $\sigma \simeq \pm 0.3 \mathrm{mag}$.).

One may question, however, whether the brightest elliptical galaxy in every cluster is intrinsically nearly the same, or, more precisely (and cogent for cosmological purposes), whether any differences in the absolute magnitudes of the brightest elliptical cluster galaxies are independent of cluster richness or distance. E. Scott (1957) has shown that under certain circumstances the richnesses and distances of clusters selected 
for observational study should be correlated. If this 'Scott effect' is present, it has important cosmological consequences.

A preliminary study by Peterson (1970) of some 48 clusters (which largely overlap Sandage's sample of clusters) indicates that indeed there may be a positive correlation between cluster richnesses and the absolute magnitudes of their brightest galaxies. Because the Virgo cluster is not a rich cluster (compared to most of those well-studied systems of cosmologically-significant distances), Peterson's results cast doubt on the validity of the magnitude of the brightest galaxy in the Virgo cluster as a calibrator for the extragalactic distance scale.

Moreover, W. W. Morgan and his collaborators (Matthews et al., 1964; Morgan and Lesh, 1965) have called attention to the presence of supergiant elliptical $(c D)$ galaxies in some, but not all, clusters, and Morgan and Bautz (1970) have suggested a classification scheme based on the luminosity of the brightest elliptical galaxy in a cluster. An example mentioned by the latter of a cluster containing a supergiant elliptical is A2199 (centered on NGC 6166); an example of a cluster whose brightest elliptical galaxies do not appear to be intrinsically overluminous is A2065 (Corona Borealis). These rather obvious differences between clusters, as regards their brightest members, cast further suspicion on the security of using the brightest cluster member as a distance indicator.

\section{Cluster Luminosity Functions as Distance Indicators}

Another approach to obtaining relative distances of clusters is through comparisons of their luminosity functions. For some years the writer has been investigating the luminosity functions of rich clusters, one purpose of which has been to see if they contain features that might be useful for distance indicators (Abell, 1962, 1965). His studies include several rich clusters that contain galaxies that are almost entirely of the E and S0 types. In each case, the integrated luminosity function rises sharply at the bright end, and rather abruptly levels off, rising more slowly at fainter magnitudes. The logarithm of the number of galaxies brighter than apparent magnitude $m$ is approximately proportional to $0.8 \mathrm{~m}$ for the interval of the first 2 or 3 magnitudes, until a magnitude is reached that the writer denotes by $m^{*}$; subsequently, the logarithmic function is approximately proportional to $0.25 \mathrm{~m}$ (see the plot of the luminosity function for the Coma cluster in Figure 2). This result is consistent, at least for all but the brightest magnitudes, with the cluster luminosity function derived by Zwicky (1957) from an analysis of the diameters of clusters with various numbers of visible galaxies, and, incidentally, predicted by Zwicky earlier from quasi-statistical mechanical arguments (1942). More recently, Rood (1969) has found a similar luminosity function for the galaxies in the central part of the Coma cluster, and Holmberg (1969) has derived an almost identical luminosity function from Zwicky's published magnitudes of galaxies in the Virgo cluster.

It is tempting to suggest that $m^{*}$, which marks a maximum in the bright end of the differential cluster luminosity function, might represent a mass (or dimension) which 
corresponds to some characteristic parameter relevant to the formation of galaxies in clusters, in which case, $m^{*}$ might be expected to occur at the same absolute magnitude in all rich clusters. If such were the case, $m^{*}$ should be a useful criterion of distance for those clusters containing elliptical galaxies.

Indeed, it does happen that a plot of $\log z$ vs $m^{*}$ for those rich clusters of known redshift for which the writer has obtained luminosity functions does show very small scatter (Figure 1). The points in Figure 1 referring to the Virgo cluster are discussed below.

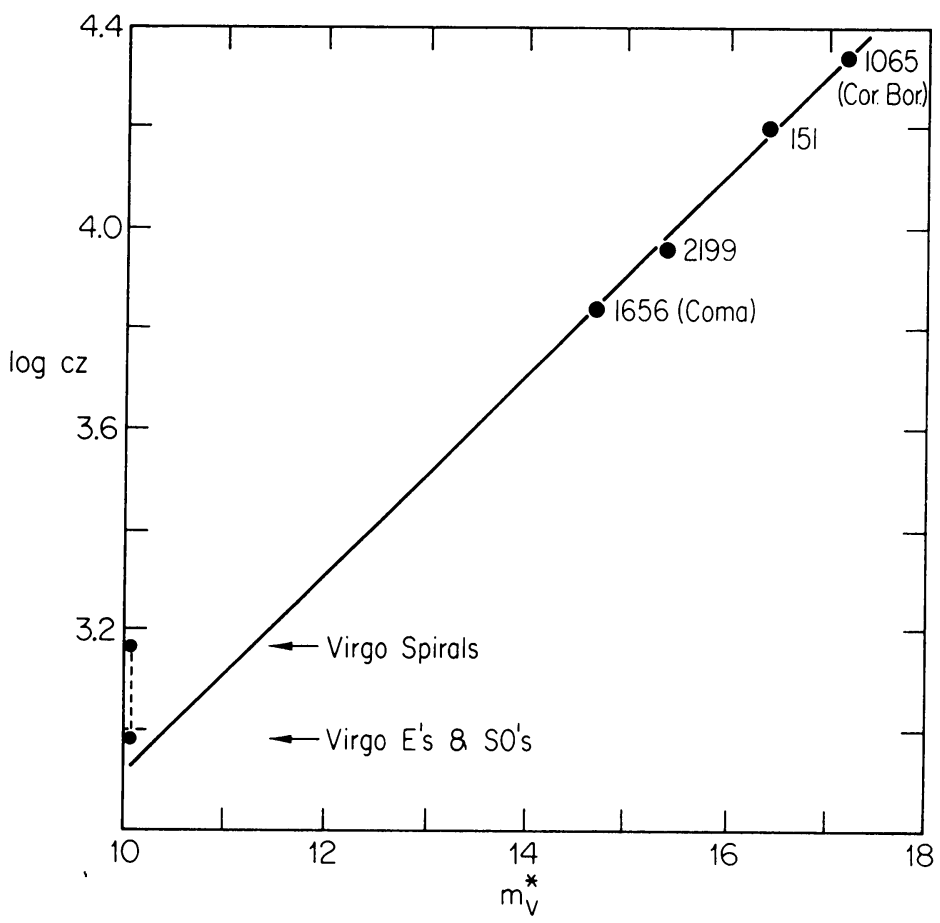

Fig. 1. Logarithms of the radial velocities vs $m_{p v}{ }^{*}$ for those clusters (numbers from the Abell cluster catalogue) of measured radial velocity for which the luminosity functions have been investigated. The solid line has the theoretically expected slope of 0.2 .

Because the Virgo cluster is the only one for which we can at present estimate a distance from observations of well-understood distance indicators, the writer, in collaboration with Mr S. Eastmond $(1968,1970)$, has carried out photometry to obtain the luminosity function of the elliptical galaxies in the Virgo cluster. The magnitudes have been obtained by a procedure of photoelectrically-calibrated extrafocal photographic photometry described by Abell and Mihalas (1966). All elliptical galaxies brighter than $m_{p v}=16.0$ in a field covering 168 square degrees of the Virgo cluster have been measured on in-focus and extra-focal photographs taken with the 48-in. Palomar Schmidt. The integrated luminosity function obtained for these galaxies is shown as the solid histogram in Figure 2. 


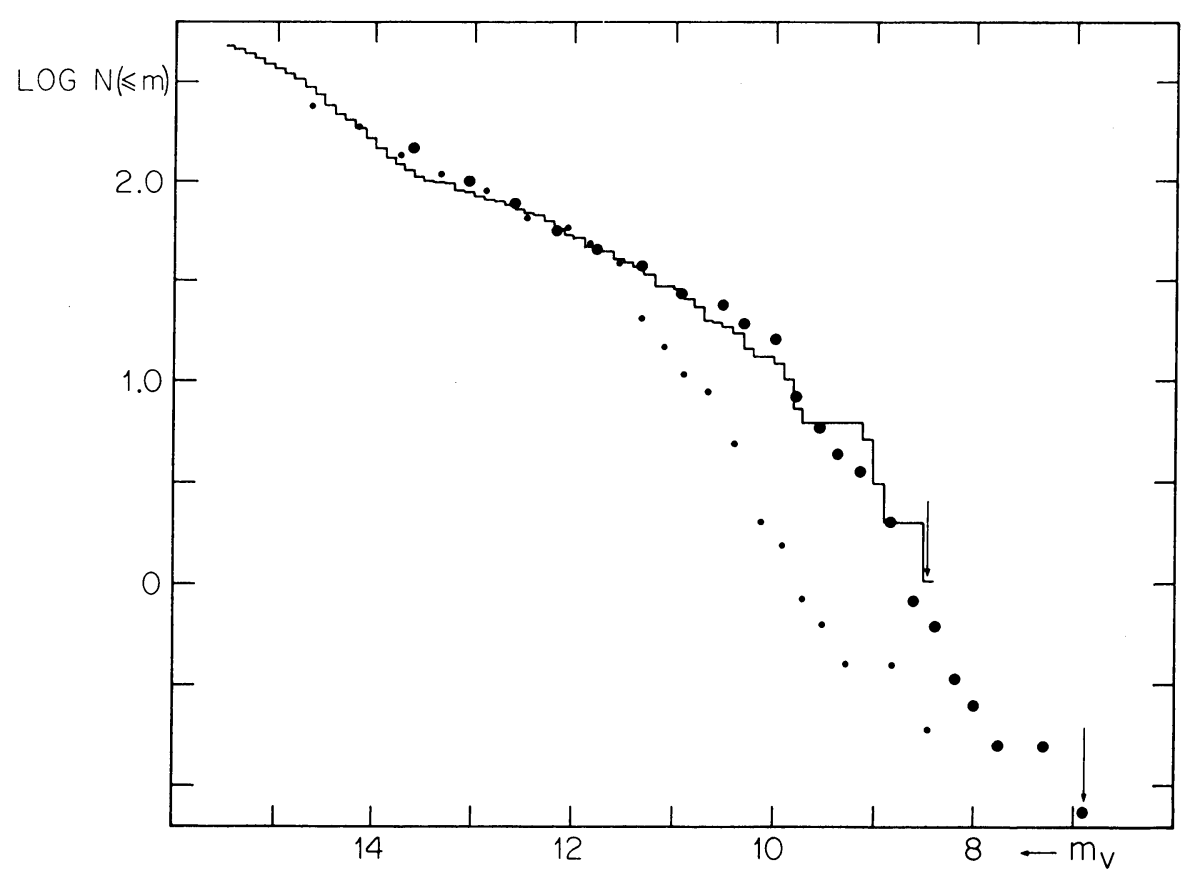

Fig. 2. Logarithm of the numbers of galaxies brighter than apparent photovisual magnitude $m_{p v}$ vs $m_{p v}$ (solid histogram) in the central 168 square degrees of the Virgo cluster. Large filled circles: same data for the Coma cluster, shifted to match the histogram for the Virgo cluster. Small filled circles: same data for the Coma cluster, shifted to match its brightest galaxy to that of the Virgo cluster.

Although many elliptical galaxies have been measured, the writer believes the Virgo cluster to be very much poorer in membership than, say, the Coma cluster (A1656) - more typical of the rich clusters investigated previously. Consequently, the number of intrinsically bright galaxies (brighter than $m^{*}$ ) in the Virgo cluster might be expected to be much less than in the Coma cluster. This expectation is consistent with the luminosity function obtained from the Virgo cluster photometry. The luminosity function for the Coma cluster is also shown, for comparison, as large round symbols, in Figure 2. The latter has been shifted horizontally and vertically to fit, as nearly as possible, the function for the Virgo cluster. The vertical shift corresponds to the difference in intrinsic richness between the two clusters. We see from the figure that the luminosity functions of the two clusters appear to be quite similar; that for the Virgo cluster is based on comparatively few galaxies brighter than $m^{*}$ (about 20) and must, therefore, be regarded as somewhat poorly determined, but nevertheless it matches well that for the Coma cluster. The difference in richness of the two clusters (vertical shift) is nearly an order of magnitude.

The horizontal shift required to match the luminosity functions requires a rather large difference between the absolute magnitudes of the brightest elliptical galaxies in the two clusters (indicated by arrows in Figure 2). On the other hand, for the brightest cluster ellipticals to be intrinsically the same in luminosity, the Coma luminosity 
function would have to be shifted more than $1.5 \mathrm{mag}$. to the left (small dots); in this case, the two luminosity functions are quite dissimilar.

If matching luminosity functions is the appropriate way to obtain relative distances of the Virgo and Coma clusters, they differ in distance modulus by 4.7 mag. In the Coma cluster, $m_{p v}^{*}=14.7$; according to the fit in Figure 2, for the Virgo cluster, $m_{p v}^{*}=10.0$. On the other hand, if (following Sandage, 1968) the brightest galaxy in the Virgo cluster is the same in absolute magnitude as the mean of the brightest galaxies in the more remote rich clusters investigated by him (and, with the assumption that their distances are proportional to their redshifts), the difference in moduli is 3.9 mag.

It must be emphasized here that we are not suggesting errors in photometry by any of the various observers. In fact, our photometry in the Coma cluster agrees well with

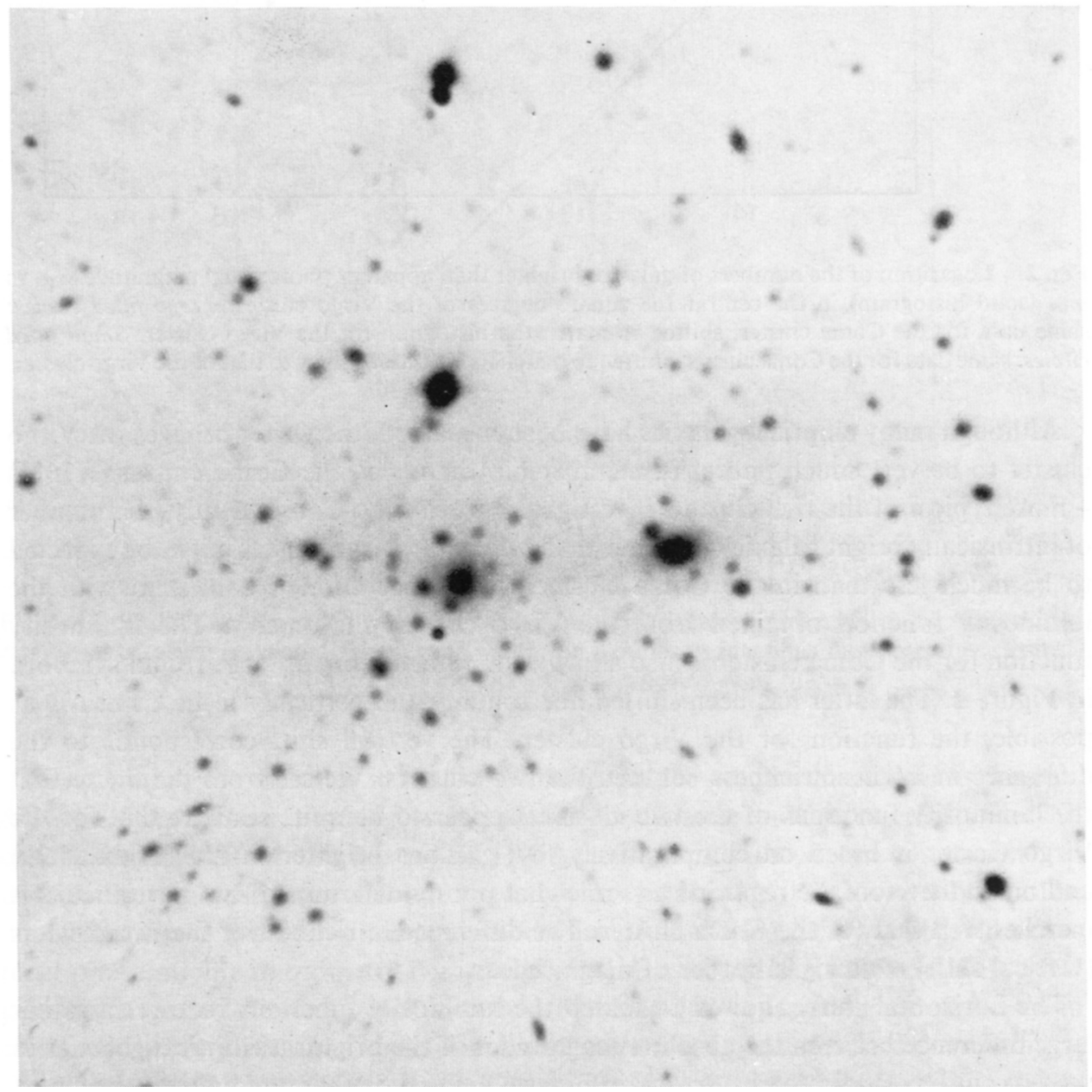

Fig. 3. The Coma cluster photographed with the 48-in. Palomar Schmidt. 
that obtained from photoelectric observations by Sandage (unpublished), and our photometry in the Virgo cluster is in good agreement with that of Holmberg (1958). The differences between the relative distance found by Sandage and that suggested here is not due to different photometric results, but rather to the interpretation of the significance of the magnitude of the brightest cluster galaxy.

Suppose, tentatively, the change in slope of the logarithmic integrated luminosity function of the Virgo cluster yields a value of $m^{*}$ that is comparable (intrinsically) to $m^{*}$ for more remote clusters; then we can plot the Virgo cluster on Figure 1 . The two points for the cluster correspond to the same modulus but with the redshifts averaged separately for the elliptical (and S0) and for the spiral galaxies. At least for the ellipticals we see that a very large deviation from a linear redshift-distance relation, even for the Virgo cluster, is not indicated.

\section{Interpretation of the Data}

The relative distances of clusters of galaxies, as we have seen, may be determined by assuming that the brightest cluster galaxy is the same in every cluster. The assump-

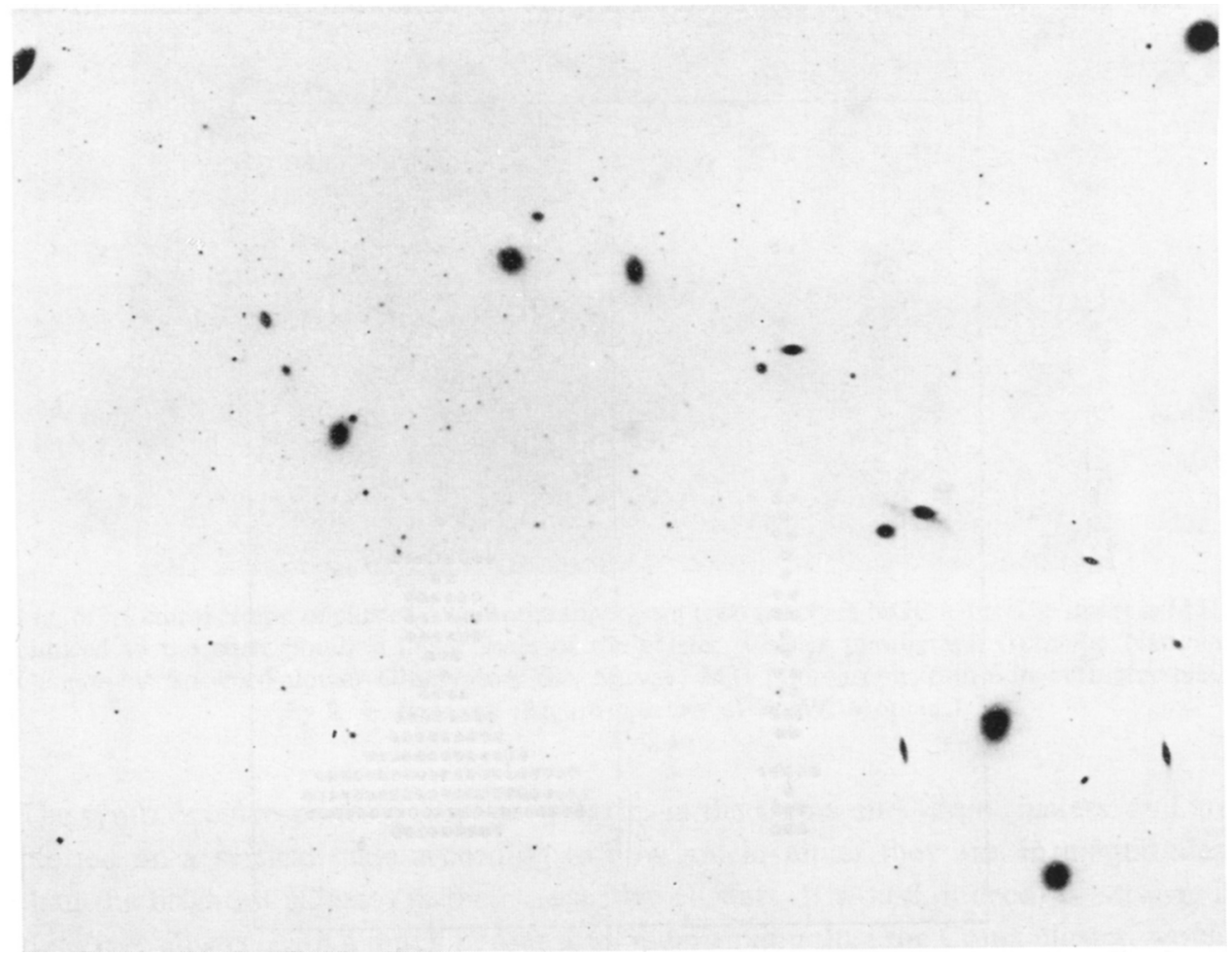

Fig. 4. The central region of the Virgo cluster photographed with the 48-in. Palomar Schmidt. M87 is in the upper right. 
tion is not strictly valid, of course, because there is an observed scatter in the absolute magnitudes of the first-ranked galaxies. They could be used as distance indicators in a statistical sense if there is no dependence of the luminosity of the brightest galaxy on cluster richness, or on some other parameter. But even if the absolute magnitude of the first-ranked galaxy had a known statistical distribution about some mean, one should exercise caution in calibrating the extragalactic distance scale with one object - the Virgo cluster. Comparison of luminosity functions suggests that the brightest Virgo galaxy, in fact, might be particularly inappropriate for the calibration.

Specifically, we must ask whether it is reasonable to assume that the brightest galaxy in the Virgo cluster (NGC 4472) can differ in absolute magnitude by more than $1.5 \mathrm{mag}$. from that of the brightest galaxy in the Coma cluster. A comparison of Figures 3 and 4 suggests that it is, indeed, reasonable. Figure 3 shows the Coma cluster. The brightest two galaxies are NGC $4884(=4889)$ and 4872 , both of which stand out nearly a full magnitude above the other galaxies in the cluster. In contrast, Figure 4 shows the central region of the Virgo cluster. The brightest galaxy, NGC 4472, is just off the field, but it differs by only a few hundredths of a magnitude from M87 (top right in Figure 4). It is obvious that M87 is not substantially brighter than other large ellipticals in the cluster, nor than spirals in the region of the cluster center.

The same comparison is exhibited in Figure 5, somewhat more quantitatively.

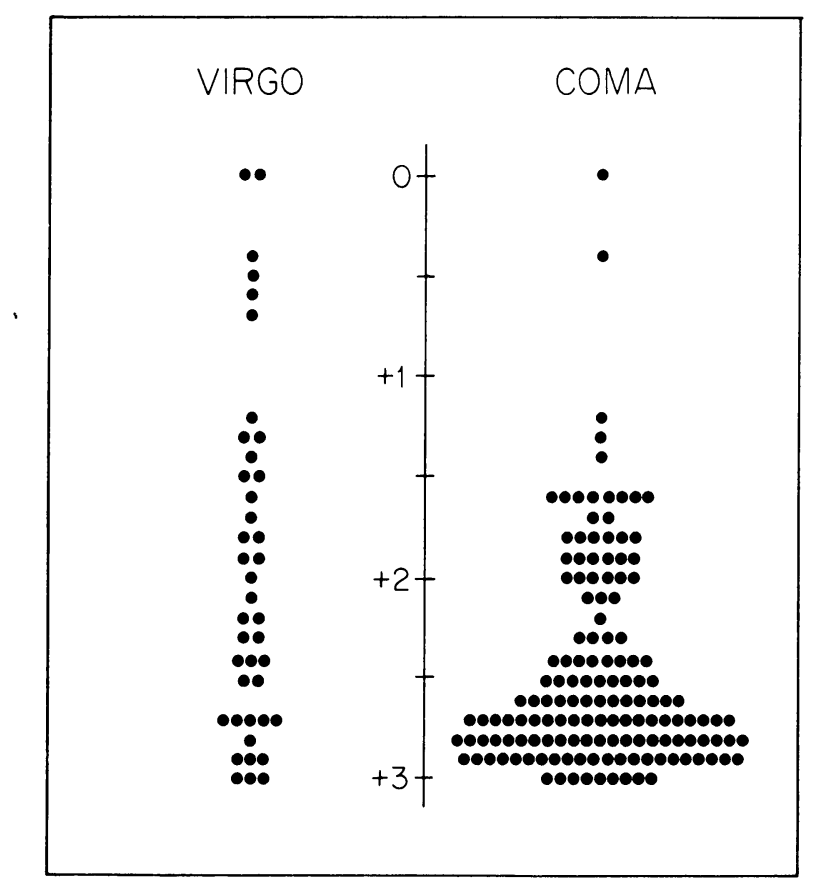

Fig. 5. The distribution in magnitudes of the brightest elliptical galaxies in the Coma and Virgo clusters. Each symbol represents a galaxy, and is plotted according to how much fainter (in photovisual magnitudes) it is than the brightest elliptical galaxy in its respective cluster. 


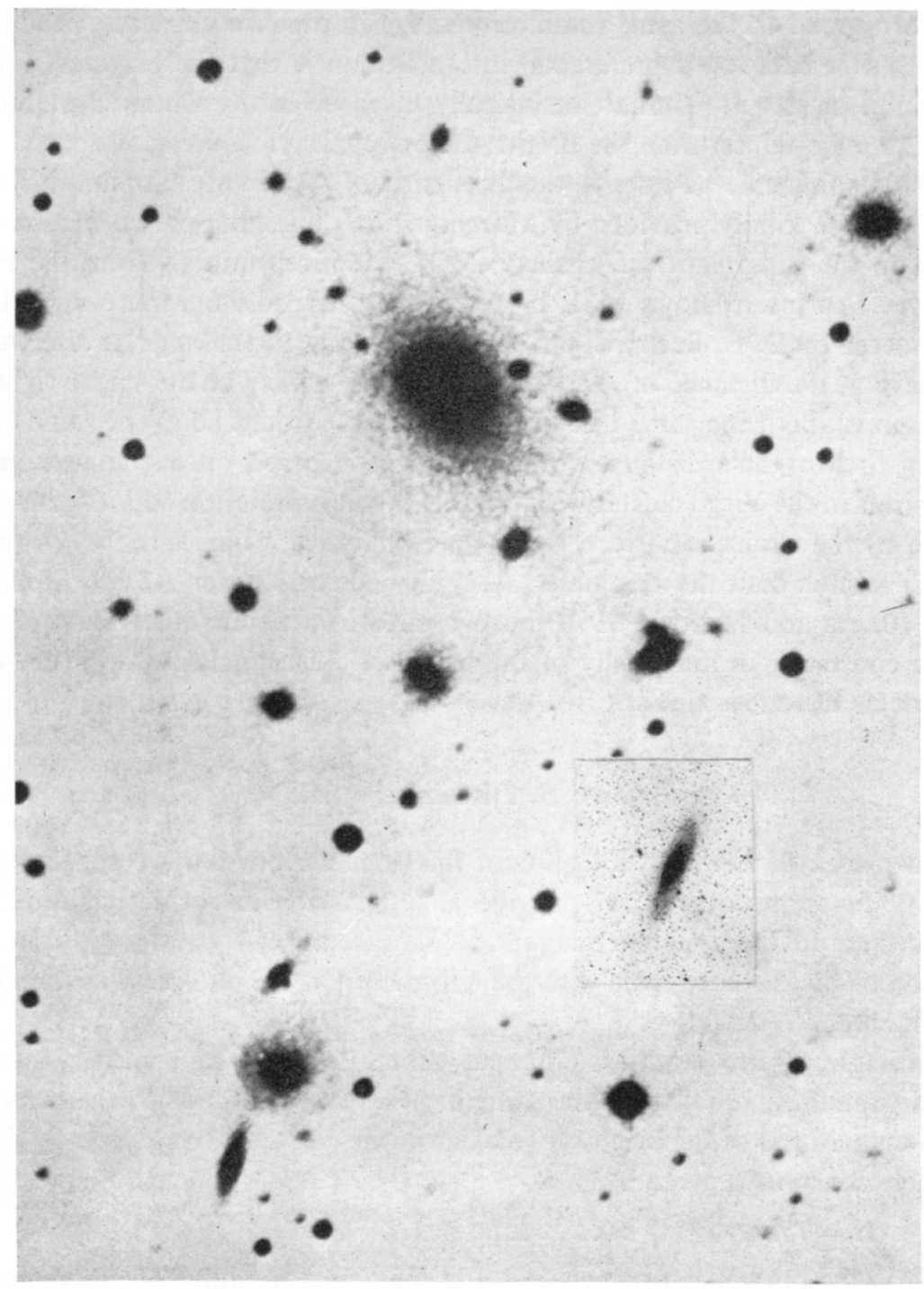

Fig. 6. Central region of cluster A2199; the supergiant $(c D)$ galaxy is NGC 6166. The insert is M31, reduced to the corresponding linear scale of the cluster. Cluster photograph from the National Geographic Society-Palomar Observatory Sky Survey; M31 photograph from 6-in. refractor plate by E. E. Barnard. (Figure courtesy of W. W. Morgan.)

The symbols correspond to elliptical galaxies in the Coma and Virgo clusters, and are plotted on a vertical scale according to how much fainter they are, in magnitudes, than the brightest galaxies in their respective clusters. It would, indeed, be strange if the Virgo cluster, with a much poorer total membership than the Coma cluster, would have 12 galaxies in the range of the brightest 1.5 magnitudes and the Coma cluster only 5 , if, in fact, the brightest galaxies were the same so that this magnitude range 
would correspond to the same luminosity range in the two clusters. An alternative (and the writer believes, a preferable) interpretation is that the brightest 6 galaxies in the Virgo cluster are similar intrinsically to those in the Coma cluster between 1.0 and 2.0 mag. fainter than the brightest Coma galaxy.

As a final comment, we refer to the discussion of W. W. Morgan and his collaborators. Figure 6, kindly provided by Morgan, shows the inner part of cluster A2199, centered on the supergiant $(c D)$ galaxy, NGC 6166, reproduced from the Palomar Sky Survey. An insert shows M31, reproduced from a photograph obtained with a 6 in. refractor by E. E. Barnard, but reduced in scale to the angular size that M31 would have at the distance of A2199. The elliptical galaxy at the upper right of the photograph is about the same in appearance as M87 would be at the same distance, according to Morgan's distance criterion, which is based on a comparison of the bright spirals in the Virgo cluster with M31. If the interpretation of Morgan, and that suggested by the writer, is correct, the brightest elliptical galaxies in the Virgo cluster are much fainter than the brightest galaxy in at least Cluster A2199. Similar conclusions (Bautz and Morgan, 1970) have been drawn about other clusters as well. Thus the constancy in luminosity of the brightest cluster galaxies, even to within a magnitude, is in serious doubt.

\section{Discussion}

The data presented here do not allow a final conclusion, but we suggest that the luminosity functions of clusters provide a better criterion for determining relative distances than do the apparent magnitudes of the brightest cluster galaxies. In the calibration of the distance scale with the Virgo cluster, the choice of criterion makes a substantial difference.

For example, if the brightest elliptical galaxy in the Virgo cluster is assumed (following Sandage, 1968) to be the same in absolute magnitude as the mean of the absolute magnitudes of the brightest galaxies in the clusters investigated by Sandage, the Hubble constant is given by

$$
H=75 \times 10^{0.2 \Delta m} \mathrm{~km} \mathrm{~s}^{-1} \mathrm{Mpc}^{-1}
$$

where $\Delta m$ is the difference between 31.1 and the actual modulus of the Virgo cluster ellipticals. On the other hand, if the luminosity function for the Virgo cluster found by Abell and Eastmond is fit to that of more remote clusters, as described in Section 3 and Figure 2,

$$
H=47 \times 10^{0.2 \Delta m} \mathrm{~km} \mathrm{~s}^{-1} \mathrm{Mpc}^{-1} .
$$

In summary, the Hubble constant should be regarded as very uncertain; in particular, a value of $H$ below $50 \mathrm{~km} \mathrm{~s}^{-1} \mathrm{Mpc}^{-1}$ can not at present be ruled out.

\section{Acknowledgements}

The writer wishes to thank the Director of the Hale Observatories for permission 
to use the 48-in. Schmidt telescope at Palomar, and the 100-in. and 60-in. telescopes on Mount Wilson, where he has obtained the photographic and photoelectric observations referred to in this note. He is also grateful for discussions with W. W. Morgan, and the latter's permission to reproduce Figure 6. The original research referred to here has been supported in large part by Contract Number N00014-67-C-0197 with the Office of Naval Research.

\section{References}

Abell, G. O.: 1958, Astrophys. J. Suppl. Ser. 3, 211.

Abell, G. O.: 1961, Astron. J. 66, 607.

Abell, G. O.: 1962, in G. C. McVittie (ed.), Problems of Extragalactic Research, New York, Macmillan Co., p. 213.

Abell, G. O.: 1965, Ann. Rev. Astron. Astrophys. 3, 1.

Abell, G. O. and Eastmond, S.: 1968, Astron. J. 73, S161.

Abell, G. O. and Eastmond, S.: 1970, Bull. Am. Astron. Soc. 2, 179.

Abell, G. O. and Mihalas, D. M.: 1966, Astron. J. 71, 635.

Bautz, L. P. and Morgan, W. W.: 1970, Astrophys. J. Letters 162, L149.

Carpenter, R. L.: 1961, Publ. Astron. Soc. Pacific 73, 324.

Holmberg, E.: 1958, Medd. Lunds Astron. Obs. Ser. II, No. 136.

Holmberg, E.: 1962, in G. C. McVittie (ed.), Problems of Extragalactic Research, New York, Macmillan Co., p. 401.

Holmberg, E.: 1969, Ark. Astron. 5 (No. 20), 305.

Matthews, T. A., Morgan, W. W., and Schmidt, M.: 1964, Astrophys. J. 140, 35.

Morgan, W. W. and Lesh, J. R.: 1965, Astrophys. J. 142, 1364.

Peterson, B. A.: 1970, Astrophys. J. 159, 333.

Racine, R.: 1968, J. Roy. Astron. Soc. Can. 62, 367.

Rood, H. J.: 1969, Astrophys. J. 158, 657.

Sandage, A. R.: 1968, Astrophys. J. Letters 152, L149.

Scott, E. L.: 1957, Astron. J. 62, 248.

Vaucouleurs, G. de: 1953, Astron. J. 58, 30.

Vaucouleurs, G. de: 1956, in A. Beer (ed.), Vistas in Astronomy 2, Pergamon Press, London and New York, p. 1584.

Vaucouleurs, G. de: 1958, Astron. J. 63, 253.

Vaucouleurs, G. de: 1961, Astrophys. J. Suppl. 6, 213.

Vaucouleurs, G. de: 1970, Astrophys. J. 159, 435.

Zwicky, F.: 1942, Phys. Rev. 61, 489.

Zwicky, F.: 1957, Morphological Astronomy, Springer Verlag, Berlin p. 171.

\section{Discussion}

Peach: How do you make field corrections in forming your luminosity functions and more positively, is the position of the discontinuity in apparent magnitude dependent on this correction?

Abell: (replying to unrecorded comment by Peach) There is no discrepancy over photometry. My magnitudes for the bright Coma galaxies are in excellent agreement with those measured photoelectrically by Sandage. I am not aware that Sandage has independent photometry of Virgo galaxies that is published (other than his corrected version of Pettit's early photometry), but my Virgo photometry agrees very well with that by Holmberg. The discrepancy is not in photometry, but arises from different assumptions about the relative distances of the clusters, that is, the discrepancies are in absolute, not apparent magnitudes.

Now to your question: In the clusters which I am comparing with the Virgo cluster (A151, 1656, 2065, and 2199) the magnitudes at which the logarithmic integrated luminosity function changes slope are all bright enough that the way the background galaxies are taken into account can have no 
effect. The background (or 'field') galaxies become significant only at magnitudes fainter than $m_{p v}=17$, and I have fitted the luminosity functions together at brighter magnitudes.

Oort: Referring to Abell's interesting suggestion that the brightest galaxies in the Virgo cluster might be $1^{m} .5$ fainter than the more outstanding brightest members of clusters like that in Coma, one should stress that the Virgo cluster is the only one where we have absolute determinations of the distance. So I would think that the value of $H$ might be fundamentally based on the Virgo cluster; that is, unless the Virgo cluster or the local group has a considerable peculiar velocity, which seems improbable.

Abell: Actually, the mean radial velocity of the elliptical galaxies in the Virgo cluster, if my relative distances are correct, is only slightly greater than would be predicted from the lower value of the Hubble constant. The spirals in Virgo have a mean radial velocity some $400 \mathrm{~km} \mathrm{~s}^{-1}$ greater, but this discrepancy between the mean redshifts of spirals and ellipticals remains to be explained, regardless of the distance scale. 